\title{
Perspective of Human Condensins Involved in Colorectal Cancer
}

\author{
Hongzhen Wang ${ }^{1 *}$, Yao Chen ${ }^{1}$, Dawei Yang ${ }^{2}$ and Liang $\mathrm{Ma}^{2 *}$ \\ ${ }^{1}$ School of Life Sciences, Jilin Normal University, Siping, China, ${ }^{2}$ The Department of General Surgery, The Central People's \\ Hospital of Siping City, Siping, China
}

\section{OPEN ACCESS}

Edited by:

Ester Pagano,

University of Naples Federico II, Italy

Reviewed by:

Haibin Tong,

Wenzhou University, China

Yongming Wang,

Fudan University, China

Jianzhou Yang,

Changzhi Medical College, China

ChunMei Zhang,

Jilin University, China

Zhiwu Wang,

Tangshan People's Hospital, China Xiaomeng $\mathrm{Li}$,

Northeast Normal University, China

*Correspondence:

Hongzhen Wang

wanghz2018@foxmail.com

Liang Ma

marvin201607@163.com

Specialty section:

This article was submitted to

Gastrointestinal and Hepatic

Pharmacology,

a section of the journal

Frontiers in Pharmacology

Received: 06 February 2021

Accepted: 19 August 2021

Published: 07 September 2021

Citation:

Wang $H$, Chen $Y$, Yang $D$ and $M a L$

(2021) Perspective of Human

Condensins Involved in

Colorectal Cancer.

Front. Pharmacol. 12:664982.

doi: 10.3389/fphar.2021.664982
Although many important roles are played by human condesins in condensation and segregation of mitotic chromosomes, what roles of human condensins play in colorectal cancer are still unclear at present. Recently, abnormal expressions of all eight subunits of human condensins have been found in colorectal cancer and they are expected to become potential biomarkers and therapeutic targets for colorectal cancer in the future. However, there are still no reviews on the significance of abnormal expression of human condensin subunits and colorectal cancer until now. Based on a brief introduction to the discovery and composition of human condensins, the review summarized all abnormally expressed human subunits found in colorectal cancer based on publicly published papers. Moreover, Perspective of application on abnormally expressed human subunits in colorectal cancer is further reviewed.

Keywords: condensin, human condensin, structure of chromosomes protein, abnormal expression, colorecal cancer

\section{INTRODUCTION}

Nowadays colorectal cancer (CRC) is the second leading cause of cancer mortality and the third most commonly diagnosed cancer worldwide (Bray et al., 2018). With the rapid development of the economy, life expectancy correlated positively with the incidence and mortality of CRC both in men and women in China (Gu et al., 2018). Although there has been a clear treatment method for the early colorectal cancer with general surgery and adjuvant chemotherapy, many colorectal cancer patients are in the late stage when they were firstly identified because of the lack of effective biomarkers in early screening of colorectal cancer and they are hard to cure. Therefore, it is of great significance to strengthen the study of molecular mechanism of colorectal cancer and develop effective biomarkers and therapeutic targets for early screening and diagnosis of colorectal cancer. The identification of abnormal expression proteins of colon cancer is pivotal for early detection of colorectal cancer and effective development of biomarkers and therapeutic targets.

Recently, all eight human condensin subunits have been found abnormally expressed in colorectal cancer and they are expected to become potential biomarkers and therapeutic targets for colorectal cancer in the future (Dávalos et al., 2012; Shiheido et al., 2012; Tokunaga et al., 2013; Feng et al., 2014; Je et al., 2014; Jinushi et al., 2014; Yin et al., 2017; Baergen et al., 2019; Montero et al., 2020; Yeh et al., 2020). Therefore, based on the brief introduction of the discovery and composition of human

Abbreviations: CRC, colorectal cancer; SMC, structure maintenance of chromoses; hCAP, human chromosome associate polypeptide; NCAP, non-SMC condensin I complex subunit; MSI-H, high microsatellite instability; GRID2, glutamate ionotropic receptor delta type subunit 2; CBLN1, cerebellin 1 precursor; POU2F1, TF POU class 2 homeobox 1; HPGDS, hematopoietic prostaglandin D synthase. 
TABLE 1 | All eight subunits of human condensins involved in colorectal cancer

Human condensin subunits involved in

colorectal cancer

hCAP-G2(NCAPG2)

All eight human condensin subunits

$\begin{array}{ll}\text { SMC2(hCAP-E) } & \text { unclear/overexpression } \\ & \text { frameshift mutation/null } \\ & \text { not mutated/reduced expression } \\ & \text { unclear/unclear }\end{array}$

unclear/unclear

Mutated gene/protein expression

Number of related literature and published year

unclear/expression of hCAP-G2 is affected by binding of Q15 or Q15 and MIP-2A

gene copy number alterations include deletions, small-scale gains and/or largescale amplifications/reduced expression

SMC4(hCAP-C)

unclear/overexpression

unclear/overexpression

unclear/overexpression

unclear/upregulated

missense and frameshift mutation/overexpression
Shiheido et al., 2012

Baergen et al., 2019

Dávalos et al., 2012

Je et al., 2014

Montero et al., 2020

Yeh et al., 2020

Dávalos et al., 2012

Feng et al., 2014

Jinushi et al., 2014

Zhang et al., 2021
Tokunaga et al., 2013

hCAP-H(NCAPH)

Yin et al., 2017 condensins, this paper reviews the research progress and application perspective of all eight human condensin subunits in colorectal cancer to offer beneficial reference for related research.

\section{Discovery and Composition of Human Condensins}

In 2001, human condensin I was firstly purified based on the study of human ortholog of frog condensin from HeLa nuclear extracts (Hirano and Mitchison, 1994; Schmiesing et al., 2000; Kimura et al., 2001). In 2003, Ono et al. (2003) discovered another condensin complex in HeLa nuclear extracts and the two kinds of condesins in human cells are termed as condensin I and II respectively (Kimura et al., 2001).

Both of human condensins are composed of five evolutionally conserved subunits. SMC2 (also named as human chromosome associate polypeptide E, hCAP-E) and SMC4(hCAP-C)are formed heterodimer and shared by the two kinds of human condesins (Schmiesing et al., 1998; Kimura et al., 2001; Ono et al., 2003). The other three non-SMC subunits are hCAP-D2 (NCAPD2), hCAP-H (NCAPH) and hCAP-G (NCAPG) in human condensin I and hCAP-D3 (NCAPD3), hCAP-H2 (NCAPH2) and hCAP-G2 (NCAPG2) in human condensin II (Kimura et al., 2001; Ono et al., 2003). Although the two kinds of human condensins have similar composition and alphabetic structure, they play different roles in the chromosome dynamics during the cell cycle (Hirota et al., 2004; Ono et al., 2004; Onn et al., 2007).

\section{All Eight Subunits of Human Condensins are Abnormally Expressed in Colorectal Cancer} All eight subunits of human condensins have been reported to be abnormally expressed in colorectal cancer until now, as shown in Table 1 (Dávalos et al., 2012; Shiheido et al., 2012; Tokunaga et al., 2013; Feng et al., 2014; Je et al., 2014; Jinushi et al., 2014; Yin et al., 2017; Baergen et al., 2019; Montero et al., 2020; Yeh et al., 2020; Zhang et al., 2021).

Firstly, expression of hCAP-G2, a non-SMC subunit of human codensin II, is affected by binding of Q15 (an anilinoquinazoline derivatives) and inhibiting proliferation of multiple cultured colorectal cancer cell lines (Shiheido et al., 2012). Furthermore, Q15 can also binds to MIP-2A (MBP-1 interacting protein-2A) and simultaneous targeting of hCAPG2 and MIP-2A may be a promising strategy for the treatment of intractable colorectal tumors (Tokunaga et al., 2013).

Secondly, hypomorphic expression or no expression of SMC2 (hCAP-E) is implicated in cancer pathogenesis. Concretely, SMC2 gene is frameshift mutated because of mononucleotide repeats and this causes loss of its expression in colorectal cancer with high microsatellite instability (MSI-H) (Je et al., 2014). Likewise, reduced expression of all condensin genes may drive chromosome instability (CIN) and may contribute to colorectal cancer pathogenesis (Baergen et al., 2019). Consistent with this, inhibition of tumorsphere formation in colon cancer cell lines was observed recently when SMC2 was inhibited by intracellular delivery of specific anti-SMC2 antibodies (Ab-SMC2) alone or with anticancer drug by polymeric micelles (PM) (Montero et al., 2020). On the other hand, overexpression of SMC2 has also been found in both colorectal cancer cell lines and samples from CRC patients (Dávalos et al., 2012). The difference between overexpression and low expression of SMC2 may because that it is regulated by different signaling pathways. SMC2 promoter could be driven by WNT signaling and it cause SMC2 abnormal expression correlated with $\beta$-catenin levels in CRC cell lines and clinical samples (Dávalos et al., 2012). Similarly, it is reported that the receptor glutamate ionotropic receptor delta type subunit 2 (GRID2) binds ligand cerebellin 1 precursor (CBLN1) to transmit the signal to TF POU class 2 homeobox 1 (POU2F1) through SMC2 (Yeh et al., 2020). This signal upregulates the target gene hematopoietic prostaglandin D synthase (HPGDS) and inhibits the migration and proliferation of CRC cells (Tippin et al., 2012). 
These results mentioned above suggest that dysfunction of SMC2 is involved in pathogenesis of colorectal cancer.

Thirdly, SMC4 also shows dysregulations in colorectal cancer cells. For example, overexpression of SMC4 was found in both colorectal cancer cell lines and samples from CRC patients and downregulation of SMC4 plays a suppressive role in the proliferation of colorectal cancer cells and tumor growth (Dávalos et al., 2012; Feng et al., 2014). In addition, it is demonstrated that expression of SMC4 mRNA is downregulated by miR-124-5p (also known as miR-124*) and growth of human colon adenocarcinoma cell is inhibited. Therefore low expression levels of microRNA-124-5p and overexpression of SMC4 is correlated with poor prognosis in colorectal cancer (Jinushi et al., 2014). Consistent with this, recently Zhang et al. (2021) finally identified two molecular subtypes that had been named $\mathrm{C} 1$ and $\mathrm{C} 2$ based on the cell cycle-related genes in patients with colon cancer and SMC4 were identified to be one of fifty upregulated genes as markers for C2 subtypes.

Finally, NCAPH is overexpressed in colorectal cancer cell lines comparing with normal human colonic epithelial cells. Many NCAPH mutations include missense and frameshift are identified in colorectal cancer patients (Yin et al., 2017). Of note, contrast to SMC4, the patients with NCAPH overexpression in colon cancerous tissues had a significantly better prognosis and survival rate than those with low-expression of NCAPH in tumor tissues.

\section{CONCLUSION AND FUTURE PERSPECTIVES}

Taken together, all eight human condensin genes have been found to be abnormally expressed in colorectal cancer until now. Although there are differences in different colorectal cancers with upregulation or downregulation of individual human condensin subunit, it just shows that colorectal cancer has complex signal transduction and genomic and transcriptional heterogeneity (Kyrochristos and Roukos, 2019).

At present, the identification of tumor specific targets or characteristics is of great significance for the treatment of cancer patients (Andre et al., 2014). The development of potential biomarkers to assist individualized therapy and drug discovery is becoming a new trend in the diagnosis and treatment of colorectal cancer (Zhai et al., 2017). The discovery of abnormal expression of human condensin subunits in colorectal cancer opens up a new way for the diagnosis and treatment of colorectal cancer. With the development of evidence-based colorectal cancer precision medicine, all eight condensin subunits could be potential biomarkers and therapeutic targets for colorectal cancer (Dávalos et al., 2012; Shiheido et al., 2012; Feng et al., 2014; Jinushi et al., 2014; Yin et al., 2017; Baergen et al., 2019; Yeh et al., 2020). However, the molecular mechanism of how they function in the process of tumorgenensis remains to be further explored in the future. There are three aspects worthy of attention about the future application research.
Firstly, the molecular mechanism of eight human condensin subunits involved in colorectal cancer needs further research. What roles each human condensin subunit plays in pathology of colorectal cancer are not very unclear. Whether the instability of chromosome is affected by the activity of the whole complex of human condensins or by some unknown signal transduction of each human condensin subunit is also unclear. Recently, it is reported that reduced condensin expression and function may be a significant, yet, underappreciated driver of colorectal cancer (Baergen et al., 2019). On the other hand, according to the idea that the human condensin subunits plays a role by affecting the function of the whole human condensin complex, Palou et al. (2018) proposed a cancer treatment hypothesis based on the overexpression of SMC4, a subunit of human condensin. SMC4 gene is often overexpressed in cancer cells, which exceeds the activity required for chromosome condensation in normal cells. Therefore, some inhibitors that inhibit the activity of condensin may kill cancer cells without damaging normal cells, and appropriately inhibiting the activity of ATPase of human condensin may selectively kill cancer cells without damaging normal cells.

Secondly, the microRNAs that regulate the expression of eight human condensin subunits in colorectal cancer need further research. MicroRNA can be developed as a biomarker for colorectal cancer (Ahmed, 2014; Qin and Liu, 2019; Farace et al., 2020). For example, mir-124-5p plays a role by regulating the expression of SMC4 target gene, which can be used as a biomarker in the diagnosis and treatment of colon cancer patients (Jinushi et al., 2014). The results suggest that microRNAs specifically target the other seven human condensin subunits may also be developed as potential biomarkers and therapeutic targets for colorectal cancer.

Finally, the interaction between the reported biomarkers of colorectal cancer and eight human condensin subunits in colorectal cancer need further research. Until now, multiple biomarkers for colorectal cancer have been found (Ye et al., 2016; Li et al., 2019; Erfani et al., 2020; Harvey et al., 2020; Huang et al., 2020; Lu et al., 2020). Nevertheless, it is unclear whether and/or how these biomarkers interact with the eight human condensin subunits in colorectal cancer.

Moreover, hCAP-D2(NCAPD2) and hCAPD3(NCAPD3), non-SMC subunit of human condensin I or condensin II respectively, are overexpressed in patients with ulcerative colitis and the overexpression of these two proteins is regulated by $I K K / N F-\kappa B$ signal transduction pathway to promote the release of inflammatory cytokines (Yuan et al., 2019). Similarly, as mentioned above, SMC2 is regulated by WNT signaling and it cause SMC2 abnormal expression in CRC cell lines and clinical samples (Tokunaga et al., 2013). These studies suggested that individual human codensin subunit may have a different signal transduction pathway and the same human codensin subunit may be regulated by different signal transduction pathway in colorectal cancer and related disease.

All in one, it opens up a new way for the diagnosis and treatment of colorectal cancer from the point of exploring 
novel function of human condensin subunits in colorectal cancer. Up to now, it is reported that all subunits of human condensins are involved in the tumorgenensis and can be potential biomarkers and therapeutic targets (Dávalos et al., 2012; Shiheido et al., 2012; Feng et al., 2014; Jinushi et al., 2014; Wang et al., 2018; Baergen et al., 2019; Xiao et al., 2020; Yeh et al., 2020; Zhang et al., 2020). With further research on the molecular mechanism of the eight human condensin subunits in colorectal cancer, more attention will be paid to the development and application of these subunits as potential biomarkers and therapeutic targets in the diagnosis and treatment of colorectal cancer in the foreseeable future.

\section{REFERENCES}

Ahmed, F. E. (2014). miRNA as Markers for the Diagnostic Screening of colon Cancer. Expert Rev. Anticancer Ther. 14, 463-485. doi:10.1586/ 14737140.2014.869479

Andre, F., Mardis, E., Salm, M., Soria, J. C., Siu, L. L., and Swanton, C. (2014). Prioritizing Targets for Precision Cancer Medicine. Ann. Oncol. 25, 2295-2303. doi:10.1093/annonc/mdu478

Baergen, A. K., Jeusset, L. M., Lichtensztejn, Z., and McManus, K. J. (2019). Diminished Condensin Gene Expression Drives Chromosome Instability that May Contribute to Colorectal Cancer Pathogenesis. Cancers (Basel) 11, 1066. doi: $10.3390 /$ cancers 11081066

Bray, F., Ferlay, J., Soerjomataram, I., Siegel, R. L., Torre, L. A., and Jemal, A. (2018). Global Cancer Statistics 2018: GLOBOCAN Estimates of Incidence and Mortality Worldwide for 36 Cancers in 185 Countries. CA Cancer J. Clin. 68, 394-424. doi:10.3322/caac.21492

Dávalos, V., Súarez-López, L., Castaño, J., Messent, A., Abasolo, I., Fernandez, Y., et al. (2012). Human SMC2 Protein, a Core Subunit of Human Condensin Complex, Is a Novel Transcriptional Target of the WNT Signaling Pathway and a New Therapeutic Target. J. Biol. Chem. 287, 43472-43481. doi:10.1074/ jbc.M112.428466

Erfani, M., Hosseini, S. V., Mokhtari, M., Zamani, M., Tahmasebi, K., Alizadeh Naini, M., et al. (2020). Altered ARID1A Expression in Colorectal Cancer. BMC Cancer. 20, 350. doi:10.1186/s12885-020-6706-X

Farace, C., Pisano, A., Griñan-Lison, C., Solinas, G., Jiménez, G., Serra, M., et al. (2020). Deregulation of Cancer-Stem-Cell-Associated miRNAs in Tissues and Sera of Colorectal Cancer Patients. Oncotarget 11, 116-130. doi:10.18632/ oncotarget.27411

Feng, X. D., Song, Q., Li, C. W., Chen, J., Tang, H. M., Peng, Z. H., et al. (2014). Structural Maintenance of Chromosomes 4 Is a Predictor of Survival and a Novel Therapeutic Target in Colorectal Cancer. Asian Pac. J. Cancer Prev. 15, 9459-9465. doi:10.7314/apjcp.2014.15.21.9459

Gu, X., Zheng, R., Xia, C., Zeng, H., Zhang, S., Zou, X., et al. (2018). Interactions between Life Expectancy and the Incidence and Mortality Rates of Cancer in China: a Population-Based Cluster Analysis. Cancer Commun. (Lond) 38, 44. doi:10.1186/s40880-018-0308-x

Harvey, J. B., Phan, L. H., Villarreal, O. E., and Bowser, J. L. (2020). CD73's Potential as an Immunotherapy Target in Gastrointestinal Cancers. Front. Immunol. 11, 508. doi:10.3389/fimmu.2020.00508

Hirano, T., and Mitchison, T. J. (1994). A Heterodimeric Coiled-Coil Protein Required for Mitotic Chromosome Condensation In Vitro. Cell 79, 449-458. doi:10.1016/0092-8674(94)90254-2

Hirota, T., Gerlich, D., Koch, B., Ellenberg, J., and Peters, J. M. (2004). Distinct Functions of Condensin I and II in Mitotic Chromosome Assembly. J. Cell Sci. 117, 6435-6445. doi:10.1242/jcs.01604

Huang, K., Gao, N., Bian, D., Zhai, Q., Yang, P., Li, M., et al. (2020). Correlation between FAK and EGF-Induced EMT in Colorectal Cancer Cells. J. Oncol. 2020, 5428920. doi:10.1155/2020/5428920

Je, E. M., Yoo, N. J., and Lee, S. H. (2014). Mutational and Expressional Analysis of SMC2 Gene in Gastric and Colorectal Cancers with Microsatellite Instability. APMIS 122, 499-504. doi:10.1111/apm.12193

\section{AUTHOR CONTRIBUTIONS}

HW and LM raised the concept and design of the research. HW wrote the manuscript. YC, DY, and LM revised the article for important details. All authors have approved the submitted version.

\section{FUNDING}

This study was supported by Foundation of Jilin Educational Committee (project No. JJKH20170380KJ) and Foundation of The Central People's Hospital of Siping City.

Jinushi, T., Shibayama, Y., Kinoshita, I., Oizumi, S., Jinushi, M., Aota, T., et al. (2014). Low Expression Levels of microRNA-124-5p Correlated with Poor Prognosis in Colorectal Cancer via Targeting of SMC4. Cancer Med. 3, 1544-1552. doi:10.1002/cam4.309

Kimura, K., Cuvier, O., and Hirano, T. (2001). Chromosome Condensation by a Human Condensin Complex in Xenopus Egg Extracts. J. Biol. Chem. 276, 5417-5420. doi:10.1074/jbc.C000873200

Kyrochristos, I. D., and Roukos, D. H. (2019). Comprehensive Intra-individual Genomic and Transcriptional Heterogeneity: Evidence-Based Colorectal Cancer Precision Medicine. Cancer Treat. Rev. 80, 101894. doi:10.1016/ j.ctrv.2019.101894

Li, J., He, J., Wang, Y., Shu, Y., and Zhou, J. (2019). SMC1 Promotes Proliferation and Inhibits Apoptosis through the NF- $\mathrm{kB}$ S-ignaling P-athway in C-olorectal C-ancer. Oncol. Rep. 42, 1329-1342. doi:10.3892/or.2019.7273

Lu, Y., Kweon, S. S., Cai, Q., Tanikawa, C., Shu, X. O., Jia, W. H., et al. (2020). Identification of Novel Loci and New Risk Variant in Known Loci for Colorectal Cancer Risk in East Asians. Cancer Epidemiol. Biomarkers Prev. 29, 477-486. doi:10.1158/1055-9965.EPI-19-0755

Montero, S., Seras-Franzoso, J., Andrade, F., Martinez-Trucharte, F., VilarHernández, M., Quesada, M., et al. (2020). Intracellular Delivery of AntiSMC2 Antibodies against Cancer Stem Cells. Pharmaceutics 12, 185. doi:10.3390/pharmaceutics12020185

Onn, I., Aono, N., Hirano, M., and Hirano, T. (2007). Reconstitution and Subunit Geometry of Human Condensin Complexes. EMBO J. 26, 1024-1034. doi:10.1038/sj.emboj.7601562

Ono, T., Fang, Y., Spector, D. L., and Hirano, T. (2004). Spatial and Temporal Regulation of Condensins I and II in Mitotic Chromosome Assembly in Human Cells. Mol. Biol. Cell. 15, 3296-3308. doi:10.1091/mbc.e04-03-0242

Ono, T., Losada, A., Hirano, M., Myers, M. P., Neuwald, A. F., and Hirano, T. (2003). Differential Contributions of Condensin I and Condensin II to Mitotic Chromosome Architecture in Vertebrate Cells. Cell 115, 109-121. doi:10.1016/s0092-8674(03)00724-4

Palou, R., Dhanaraman, T., Marrakchi, R., Pascariu, M., Tyers, M., and D'Amours, D. (2018). Condensin ATPase Motifs Contribute Differentially to the Maintenance of Chromosome Morphology and Genome Stability. Plos Biol. 16, e2003980. doi:10.1371/journal.pbio.2003980

Qin, Z., and Liu, X. (2019). miR-124, a Potential Therapeutic Target in Colorectal Cancer. Onco Targets Ther. 12, 749-751. doi:10.2147/OTT.S179501

Schmiesing, J. A., Ball, A. R., Jr, Gregson, H. C., Alderton, J. M., Zhou, S., and Yokomori, K. (1998). Identification of Two Distinct Human SMC Protein Complexes Involved in Mitotic Chromosome Dynamics. Proc. Natl. Acad. Sci. U S A. 95, 12906-12911. doi:10.1073/pnas.95.22.12906

Schmiesing, J. A., Gregson, H. C., Zhou, S., and Yokomori, K. (2000). A Human Condensin Complex Containing hCAP-C-hCAP-E and CNAP1, a Homolog of Xenopus XCAP-D2, Colocalizes with Phosphorylated Histone H3 during the Early Stage of Mitotic Chromosome Condensation. Mol. Cell Biol. 20, 6996-7006. doi:10.1128/ mcb.20.18.6996-7006.2000

Shiheido, H., Naito, Y., Kimura, H., Genma, H., Takashima, H., Tokunaga, M., et al. (2012). An Anilinoquinazoline Derivative Inhibits Tumor Growth through Interaction with hCAP-G2, a Subunit of Condensin II. PLoS One 7, e44889. doi:10.1371/journal.pone.0044889 
Tippin, B. L., Levine, A. J., Materi, A. M., Song, W. L., Keku, T. O., Goodman, J. E., et al. (2012). Hematopoietic Prostaglandin D Synthase (HPGDS): a High Stability, Val187Ile Isoenzyme Common Among African Americans and its Relationship to Risk for Colorectal Cancer. Prostaglandins Other Lipid Mediat. 97, 22-28. doi:10.1016/ j.prostaglandins.2011.07.006

Tokunaga, M., Shiheido, H., Tabata, N., Sakuma-Yonemura, Y., Takashima, H., Horisawa, K., et al. (2013). MIP-2A Is a Novel Target of an Anilinoquinazoline Derivative for Inhibition of Tumour Cell Proliferation. PLoS One 8, e76774. doi:10.1371/journal.pone.0076774

Wang, H. Z., Yang, S. H., Li, G. Y., and Cao, X. (2018). Subunits of Human Condensins Are Potential Therapeutic Targets for Cancers. Cell Div. 13, 2. doi:10.1186/s13008-018-0035-3

Xiao, C., Gong, J., Jie, Y., Cao, J., Chen, Z., Li, R., et al. (2020). NCAPG Is a Promising Therapeutic Target across Different Tumor Types. Front. Pharmacol. 11, 387. doi:10.3389/fphar.2020.00387

Ye, G. D., Sun, G. B., Jiao, P., Chen, C., Liu, Q. F., Huang, X. L., et al. (2016). OVOL2, an Inhibitor of WNT Signaling, Reduces Invasive Activities of Human and Mouse Cancer Cells and Is Down-Regulated in Human Colorectal Tumors. Gastroenterology 150, 659-e16. doi:10.1053/ j.gastro.2015.11.041

Yeh, S. J., Chen, S. W., and Chen, B. S. (2020). Investigation of the Genome-wide Genetic and Epigenetic Networks for Drug Discovery Based on Systems Biology Approaches in Colorectal Cancer. Front. Genet. 11, 117. doi:10.3389/ fgene. 2020.00117

Yin, L., Jiang, L. P., Shen, Q. S., Xiong, Q. X., Zhuo, X., Zhang, L. L., et al. (2017). NCAPH Plays Important Roles in Human colon Cancer. Cell Death Dis. 8, e2680. doi:10.1038/cddis.2017.88

Yuan, C. W., Sun, X. L., Qiao, L. C., Xu, H. X., Zhu, P., Chen, H. J., et al. (2019). Non-SMC Condensin I Complex Subunit D2 and Non-SMC Condensin II Complex Subunit D3 Induces Inflammation via the IKK/NF- $\kappa B$ Pathway in
Ulcerative Colitis. World J. Gastroenterol. 25, 6813-6822. doi:10.3748/ wjg.v25.i47.6813

Zhai, Z., Yu, X., Yang, B., Zhang, Y., Zhang, L., Li, X., et al. (2017). Colorectal Cancer Heterogeneity and Targeted Therapy: Clinical Implications, Challenges and Solutions for Treatment Resistance. Semin. Cell Dev Biol. 64, 107-115. doi:10.1016/j.semcdb.2016.08.033

Zhang, Y., Liu, F., Zhang, C., Ren, M., Kuang, M., Xiao, T., et al. (2020). Non-SMC Condensin I Complex Subunit D2 Is a Prognostic Factor in Triple-Negative Breast Cancer for the Ability to Promote Cell Cycle and Enhance Invasion. Am. J. Pathol. 190, 37-47. doi:10.1016/j.ajpath.2019.09.014

Zhang, Z., Ji, M., Li, J., Wu, Q., Huang, Y., He, G., et al. (2021). Molecular Classification Based on Prognostic and Cell Cycle-Associated Genes in Patients with colon Cancer. Front. Oncol. 11, 636591. doi:10.3389/fonc.2021.636591

Conflict of Interest: The authors declare that the research was conducted in the absence of any commercial or financial relationships that could be construed as a potential conflict of interest.

Publisher's Note: All claims expressed in this article are solely those of the authors and do not necessarily represent those of their affiliated organizations, or those of the publisher, the editors and the reviewers. Any product that may be evaluated in this article, or claim that may be made by its manufacturer, is not guaranteed or endorsed by the publisher.

Copyright (c) 2021 Wang, Chen, Yang and Ma. This is an open-access article distributed under the terms of the Creative Commons Attribution License (CC $B Y$ ). The use, distribution or reproduction in other forums is permitted, provided the original author(s) and the copyright owner(s) are credited and that the original publication in this journal is cited, in accordance with accepted academic practice. No use, distribution or reproduction is permitted which does not comply with these terms. 